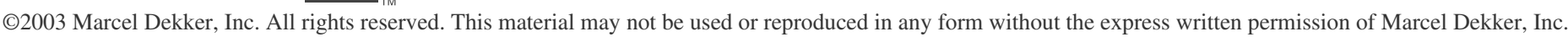

SYNTHETIC COMMUNICATIONS ${ }^{\circledR}$

Vol. 33, No. 11, pp. 1937-1941, 2003

\title{
Unexpected Nucleophilic Participation and Rearrangement of DBU in Reactions with Saccharin Derivatives
}

\author{
Philip C. Bulman Page, ${ }^{1, *}$ Hooshang Vahedi, ${ }^{1}$ \\ Donald Bethell, $^{2}$ and James V. Barkley ${ }^{2}$ \\ ${ }^{1}$ Department of Chemistry, Loughborough University, \\ Loughborough, Leicestershire, UK \\ ${ }^{2}$ Robert Robinson Laboratories, Department of \\ Chemistry, University of Liverpool, Oxford Street, \\ Liverpool, UK
}

\begin{abstract}
DBU attacks saccharin derivatives with subsequent rearrangement to give rise to 3 -[ $3^{\prime}-\left(1^{\prime \prime}\right.$-azepin-2" -onyl)propylamino]-1,2-benzisothiazole-1,1-dioxide 2 after work-up.
\end{abstract}

*Correspondence: Philip C. Bulman Page, Department of Chemistry, Loughborough University, Loughborough, Leicestershire LE11 3TU, UK; Fax: +44(0)1509 223926; E-mail: p.c.b.page@1boro.ac.uk.

1937

DOI: $10.1081 / \mathrm{SCC}-120020208$

Copyright $(2003$ by Marcel Dekker, Inc.
0039-7911 (Print); 1532-2432 (Online) www.dekker.com 
(C)2003 Marcel Dekker, Inc. All rights reserved. This material may not be used or reproduced in any form without the express written permission of Marcel Dekker, Inc.

1938

Page et al.

Key Words: DBU; Nucleophile.

In our earlier reports, ${ }^{[1]}$ it was shown that 3 -substituted-1,2-benzisothiazole-1,1-dioxides $\mathbf{1}$ derived from saccharin are able to catalyse, in sub-stoichiometric amounts, the oxidation of sulfides to sulfoxides under basic reaction conditions, as are certain other sulfonylimines. The reactions are carried out by addition of hydrogen peroxide $(30 \% \mathrm{w} / \mathrm{v}$ aqueous solution; 4.0 equiv.) to a stirred solution of base (4.0 equiv.), followed by the sulfonylimine (up to 1.0 equiv.) (Sch. 1). ${ }^{[2]}$ The imine mediators 1 were prepared according to a previously reported procedure. ${ }^{[1,3]}$ During oxidation of $p$-tolyl methyl sulfide using the mediators $\mathbf{1 a}$ and $\mathbf{1 b}$, in which the 3-substituents are $\mathrm{Cl}$ and $\mathrm{OEt}$ respectively, yields of the sulfoxide are much lower than those observed when alkyl groups occupy the 3-position. Further, methyl p-tolyl sulfoxide was obtained in substantially lower yield when using DBU as the base in the oxidation reaction than when using aqueous potassium carbonate (see Table 1). It was observed that, in the DBU reactions, an additional product could be detected by TLC analysis. This was separated from the reaction mixture, crystallized, and the structure of<smiles>[R]c1n[R5](=O)c2ccccc12</smiles>

1a $\mathrm{R}=\mathrm{Cl} ;$ 1b $\mathrm{R}=\mathrm{OEt} ; 1 \mathbf{c} \mathrm{R}=\mathrm{Me} ; \mathbf{1 d} \mathrm{R}=\mathrm{Pr}^{i} ; \mathbf{1 e} \mathrm{R}=\mathrm{Bu}^{n} ; \mathbf{1 f} \mathrm{R}=\mathrm{Bu}^{s}$ $1 \mathrm{~g} R=\mathrm{Bu}^{t} ; 1 \mathrm{~h} \mathrm{R}=\mathrm{Ph}$<smiles>CCC1CC(C)CCC1(C)C</smiles>

1j $R=$<smiles>CCC1(C)C2CCC1(C)CC2</smiles>

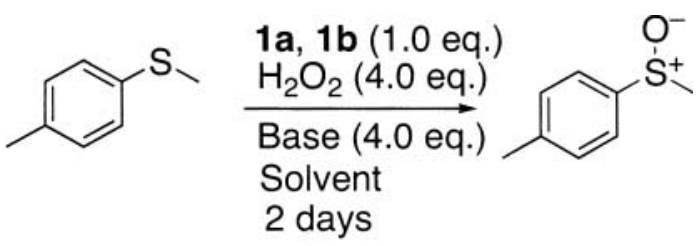

Scheme 1. 
Table 1. Oxidation of $p$-tolyl methyl sulfide catalysed by saccharin derivatives.

\begin{tabular}{lllrlcr}
\hline Mediator & Solvent & Base & $\begin{array}{c}\text { Temp. } \\
\left({ }^{\circ} \mathrm{C}\right)\end{array}$ & $\begin{array}{c}\text { Reaction } \\
\text { time }\end{array}$ & $\begin{array}{c}\text { Yield of } \\
\text { sulfoxide }(\%)\end{array}$ & $\begin{array}{r}\text { Yield of } \\
\mathbf{2}(\%)\end{array}$ \\
\hline $\mathbf{1 a}$ & $\mathrm{DCM}$ & $\mathrm{DBU}$ & -25 & 2 days & 11 & 12 \\
$\mathbf{1 b}$ & $\mathrm{DCM}$ & $\mathrm{DBU}$ & 25 & $2 \mathrm{~h}$ & & 8 \\
$\mathbf{1 b}$ & $\mathrm{DCM}$ & $\mathrm{DBU}$ & 25 & 2 days & 13 & 25 \\
$\mathbf{1 b}$ & $\mathrm{MeOH}$ & $\mathrm{K}_{2} \mathrm{CO}_{3}$ & 25 & 2 days & 33 & 0 \\
$\mathbf{1 g}$ & $\mathrm{DCM}$ & $\mathrm{DBU}$ & 25 & $2 \mathrm{~h}$ & 100 & 0 \\
\hline
\end{tabular}

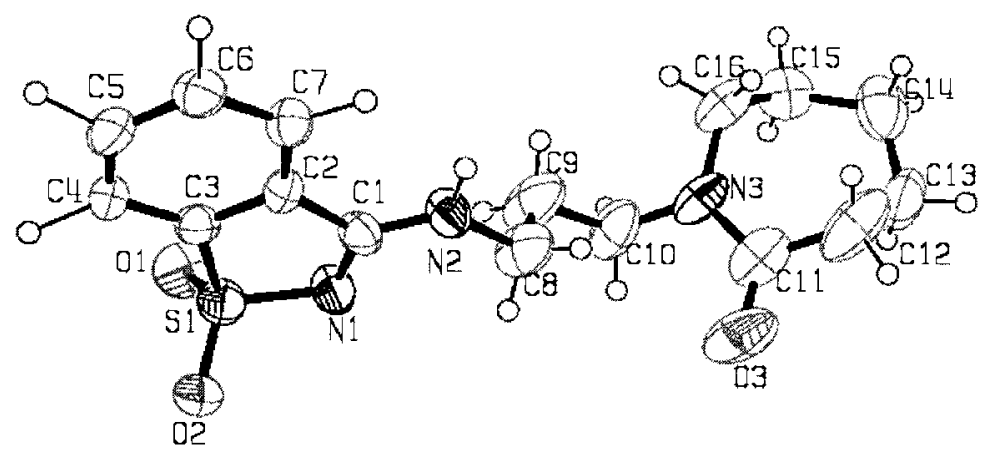

Figure 1.

the compound determined by X-ray crystallography (Fig. 1). ${ }^{\text {a }}$ To our surprise, the structure proved to be $\mathbf{2}$, containing both a saccharin residue and an $\mathrm{N}$-aminopropyl caprolactam unit, presumably derived from DBU.

The formation of compound $\mathbf{2}$ can be explained by nucleophilic attack by one nitrogen atom of the 6-membered ring of DBU at the 3-position of pseudo-saccharin ethyl ether $\mathbf{1 b}$ or pseudo-saccharyl chloride 1a, displacing ethoxide ion or chloride ion respectively. Attack on the

${ }^{a}$ Molecular formula $\quad \mathrm{C}_{16} \mathrm{H}_{21} \mathrm{~N}_{3} \mathrm{O}_{3} \mathrm{~S} \quad\left(\mathrm{M}_{\mathrm{r}}=335.42\right)$, crystal size $0.200 \times$ $0.200 \times 0.450 \mathrm{~mm}^{3}$, monoclinic, space group $\mathrm{P} 2_{1} / \mathrm{c}, a=8.463(7) \mathrm{A}^{\circ}, b=16.475$ (9) $\mathrm{A}^{\circ}, \quad c=11.835 \quad$ (7) $\mathrm{A}^{\circ}, \quad V=1646 \quad$ (2) $\mathrm{A}^{\circ 3}, \quad Z=4, \quad D=1.354 \mathrm{~g} \mathrm{~cm}^{-3}$, $\mu(\operatorname{MoK} \alpha)=2.05 \mathrm{~cm}^{-1}, F_{000}=712.2997$ Unique reflections collected on a Rigaku AFC6S sequential diffractometer, $2 \theta_{\max }=50.0^{\circ}$. The structure was solved by direct methods and $\mathrm{H}$ atoms were placed geometrically and allowed to ride on the relevant non-H atom. Refinement coverged to give $R=0.061$ and $R_{w}=0.069$. 


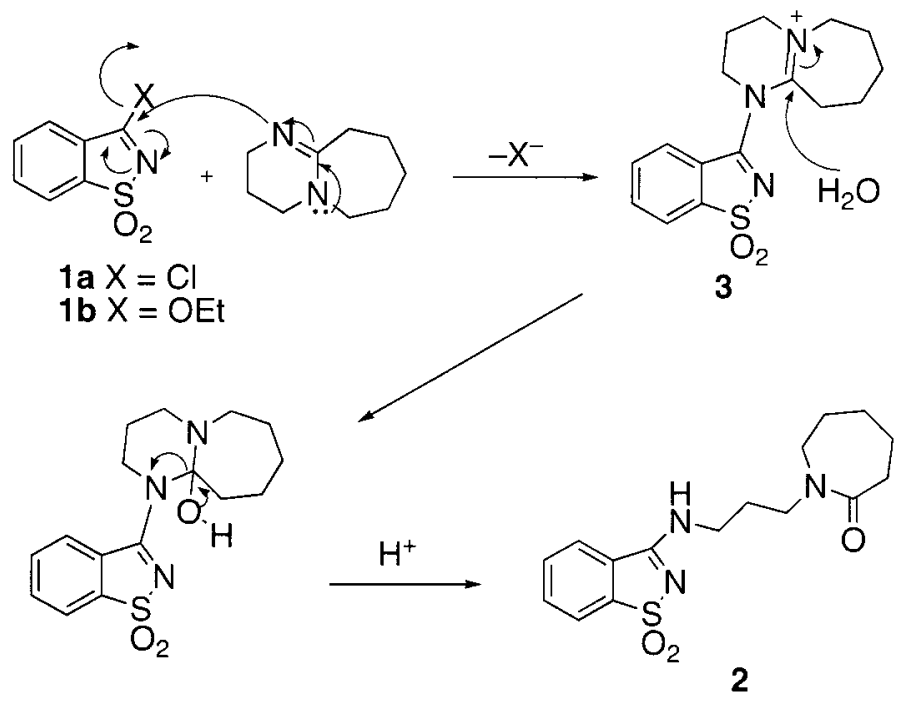

Scheme 2.

intermediate 3 by water, followed by opening of the bicyclic system, would afford compound 2 (Sch. 2). Consistent with this interpretation, compound $\mathbf{2}$ was not observed in the product mixture when alkyl saccharin derivatives $\mathbf{1 c} \mathbf{-} \mathbf{1 j}$ were used as mediators in the sulfoxidation reactions, 3-alkyl substituents not being good leaving groups. This reaction suggests that it would be wise to exercise caution when considering the use of DBU in reactions with electrophilic substrates, and particularly substrates containing good leaving groups.

\section{EXPERIMENTAL SECTION}

\section{3-[3'-(1'-Azepin-2' -onyl)propylamino]- 1,2-benzisothiazole-1,1-dioxide 2}

Hydrogen peroxide ( $30 \% \mathrm{w} / \mathrm{v}, 0.43 \mathrm{~mL}, 4$ equiv.), was added to a stirred solution of DBU $(576 \mathrm{mg}, 3.78 \mathrm{mmol})$ in dichloromethane $(10 \mathrm{~mL})$ at room temperature, followed by 3-ethoxy-1,2-benzisothiazole-1,1dioxide 1b (200 mg, $0.946 \mathrm{mmol})$. Methyl $p$-tolyl sulfide $(130 \mathrm{mg}$, $0.94 \mathrm{mmol}$ ) was added, and the reaction mixture was stirred at room temperature for $2 \mathrm{~h}$, diluted with dichloromethane $(30 \mathrm{~mL})$, washed 
with brine $(15 \mathrm{~mL})$ and saturated aqueous sodium sulfite $(20 \mathrm{~mL})$, and dried over sodium sulfate. 3-[3'-(1" -Azepin-2" -onyl)propylamino]1,2-benzisothiazole-1,1-dioxide 2 was isolated as a colourless solid by evaporation to dryness followed by flash column chromatography using ethyl acetate $\left(25 \mathrm{mg}, 8 \%\right.$ ), m.p. $212-213^{\circ} \mathrm{C}$ (from EtOH), (Found: $\mathrm{C}, 57.41 ; \mathrm{H}, 6.35 ; \mathrm{N}, 12.57 . \mathrm{C}_{16} \mathrm{H}_{21} \mathrm{~N}_{3} \mathrm{O}_{3} \mathrm{~S}$ requires $\mathrm{C}, 57.29 ; \mathrm{H}, 6.31 ; \mathrm{N}$, $12.53 \%) ; v_{\max }($ nujol $) / \mathrm{cm}^{-1} 1612,1304$ and $1152 ; \delta_{\mathrm{H}}\left(400 \mathrm{MHz} ; \mathrm{CDCl}_{3}\right)$ $1.50-1.94(8 \mathrm{H}, \mathrm{m}), 2.55(2 \mathrm{H}, \mathrm{t}, J 5.6), 3.4(2 \mathrm{H}, \mathrm{t}, J 7.4), 3.40-3.60$ $(4 \mathrm{H}, \mathrm{m}), \quad 7.50-7.70 \quad(2 \mathrm{H}, \mathrm{m}), 7.78-7.82(2 \mathrm{H}, \mathrm{m}), 8.74(1 \mathrm{H}, \mathrm{s})$; $\delta_{\mathrm{C}}\left(300 \mathrm{MHz} ; \mathrm{CDCl}_{3}\right) 23.8,26.6,28.5,30.2,37.4,39.0,45.4,50.3,122.0$, $122.1,128.8,133.1,133.4,142.9,160.0,178.6 ; \mathrm{m} / z$ (EI) $335.13036\left(\mathrm{M}^{+}\right.$, $8 \% . \mathrm{C}_{16} \mathrm{H}_{21} \mathrm{~N}_{3} \mathrm{O}_{3} \mathrm{~S}$ requires 335.13036), 140 (100) and 41 (44).

\section{ACKNOWLEDGMENT}

This investigation has enjoyed the support of Payame Noor University, Iran.

\section{REFERENCES}

1. (a) Page, P.C.B.; Bethell, D.; Stocks, P.A.; Heer, J.P.; Graham, A.E.; Vahedi, H.; Healy, M.; Collington, E.W.; Andrews, D.M. Sulfur oxidation mediated by imine derivatives. Synlett 1997, 1355; (b) Bethell, D.; Page, P.C.B.; Vahedi, H. Catalytic asymmetric oxidation of sulfides to sulfoxides mediated by chiral 3-substituted-1,2-benzisothiazole 1,1-dioxides. J. Org. Chem. 2000, 65, 6756.

2. (a) Page, P.C.B.; Heer, J.P.; Bethell, D.; Collington, E.W.; Andrews, D.M.; A new system for catalytic asymmetric oxidation of sulfides using a hydrogen peroxide based reagent. Tetrahedron Lett. 1994, 35, 9629; (b) Page, P.C.B.; Heer, J.P.; Bethell, D.; Collington, E.W.; Andrews, D.M. Highly enantioselective catalytic asymmetric oxidation of sulfides using hydrogen peroxide. Synlett 1995, 773; (c) Page, P.C.B.; Heer, J.P.; Bethell, D.; Collington, E.W.; Andrews, D.M.; Asymmetric sulfoxidation using [(3,3-dimethoxycamphoryl)sulfonyl]oxaziridine. Tetrahedron: Asymmetry 1995, 6, 2911.

3. Davis, F.A.; Towson, J.C.; Vashi, D.B.; ThimmaReddy, R.; McCauley, J.P., Jr.; Harakal, M.E.; Gosciniak, D.J. Chemistry of oxaziridines. 13. Synthesis, reactions, and properties of 3-substituted 1,2-benzisothiazole 1,1-dioxide oxides. J. Org. Chem. 1990, 55, 1254.

Received in the USA September 16, 2002 


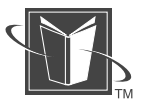

Marcel DekKer, Inc. • 270 Madison AVEnUe • NeW York, NY 10016

C일 2003 Marcel Dekker, Inc. All rights reserved. This material may not be used or reproduced in any form without the express written permission of Marcel Dekker, Inc. 